\title{
Poster: Lessons from Doing Fieldwork with Bandwidth-constrained communities Online
}

\author{
Sarina Till \\ ctill@varsitycollege.co.za \\ Independent Institute of Education \\ University of Cape Town \\ Cape Town, South Africa \\ Melissa Densmore \\ melissa.densmore@uct.ac.za \\ University of Cape Town \\ Cape Town, South Africa
}

\author{
Toshka Lauren Coleman \\ clmtos001@myuct.ac.za \\ University of Cape Town \\ South Africa, Cape Town
}

\author{
Nervo Verdezoto Dias \\ verdezotodiasn@cardiff.ac.uk \\ Cardiff University \\ Cardiff, United Kingdom
}

\section{ABSTRACT CCS CONCEPTS}

- Human-centered computing $\rightarrow$ Empirical studies in HCI.

\section{KEYWORDS}

bandwidth-constrained communities, maternal health, child health

\section{ACM Reference Format:}

Sarina Till, Toshka Lauren Coleman, Melissa Densmore, and Nervo Verdezoto Dias. 2021. Poster: Lessons from Doing Fieldwork with Bandwidthconstrained communities Online. In COMPASS '21: ACM Conference on Computing and Sustainable Societies, June 03-05, 2021, Virtual. ACM, New York, NY, USA, 4 pages. https://doi.org/10.1145/3460112.3471979

\section{INTRODUCTION AND BACKGROUND}

The COVID-19 pandemic reminded us of all the things we have taken for granted, things such as giving an old friend a hug or visiting our beloved grandparents were no longer things we just got to do. No family or industry was prepared for this rude awakening. It thus comes as no surprise that the research community was not an exception to this rule. The pandemic swiftly removed access to both remote and local communities and ceased ongoing studies with many institutions, prohibiting face-to-face in person research [2]. This left many researchers needing to find ways to work around the physical restrictions in order to engage with communities.

Traditional research methods such as workshops, in person interviews and focus groups [6] were no longer available as tools that we could readily access and conduct as part of our studies. Online platforms started gaining huge momentum [4] and researchers did what researchers do best - find new ways to conduct research, even in the toughest of circumstances. Not surprisingly many articles started to touch upon how to conduct research during the COVID-19 pandemic. These publications range from working with

Permission to make digital or hard copies of all or part of this work for personal or classroom use is granted without fee provided that copies are not made or distributed for profit or commercial advantage and that copies bear this notice and the full citation on the first page. Copyrights for components of this work owned by others than ACM must be honored. Abstracting with credit is permitted. To copy otherwise, or republish, to post on servers or to redistribute to lists, requires prior specific permission and/or a fee. Request permissions from permissions@acm.org.

COMPASS '21, June 03-05, 2021, Virtual

(C) 2021 Association for Computing Machinery.

ACM ISBN 978-1-4503-8453-7/21/06 ..\$15.00

https://doi.org/10.1145/3460112.3471979 vulnerable communities such as pregnant women [10], reaching students and conducting action research in education [11], methods for conducting clinical trials and maintaining scientific rigor [1], and in particular a list of methods for fieldwork in a pandemic was crowd-sourced [8]. Some of these papers and resources mainly reflected on their own methodologies and lessons learned so that other researchers can leverage from these experiences. However, there is currently limited research focusing on understanding and applying research methods to engage with remote and vulnerable communities especially with bandwidth constraints [7].

In 2020 we started a new project focused on digital maternal and child health, seeking to co-design potential interventions with community members. In undertaking this research during the pandemic, we drew on several resources for identifying best practices: 1) the community members themselves, 2) our experiences engaging with community members in workshops and interviews during the pandemic, 3) the expertise of researchers and practitioners familiar with community-based research, and 4) existing and developing literature in this context. In this poster we highlight what we have learned so far from community engagement activities across four communities in South Africa, and from our inter-disciplinary network of expert researchers and practitioners.

\section{METHODS}

We initially kicked off the study by conducting a literature and practice review which involved online interviews with the academic co-investigators and NGOs partners involved in the project. The review was then followed up with online interviews conducted with members from vulnerable communities in low resource settings to gain a better understanding of everyday challenges of these communities and what resources they have and needed, as well as how these communities prioritised these resources. We report on the methods our partners shared with us during the online interviews as well as the methods we used to conduct online interviews with communities based in Cape Town, Limpopo and Sweetwaters in Kwa-Zulu Natal, South Africa. The project and online research activities received ethical approval from four local institutions and one from abroad (Blinded for review). 


\subsection{Online Interviews with Researchers and Practitioners}

The multidisciplinary nature of this study had the potential to introduce disciplinary differences in the interviews and subsequent interview data. The researchers thus decided to make use of a standardized interview script. One section of this script inquired about the challenges researchers faced while conducting their research in terms of: 1) The use of Information and Communication Technology (ICT), 2) engaging with and accessing their research communities, 3) best practices in terms of ethical considerations, when working in maternal and child health research, and 4) the most appropriate and successful methods in terms of conducting community-based co-design workshops during the pandemic. The network members were emailed an invitation to schedule a 45-60-minute online interview at their convenience. This invitation contained the informed consent and participant information sheet and instructed the interviewees to return the signed consent form to the researcher before the interview was conducted. A total of thirty-four participants representing health sciences, social sciences, computer sciences and community practitioners participated. Twenty-eight participants took part in the interview process. Of these participants six represented social sciences, seven represented health sciences, nine represented computer science and a final 6 were practitioners. The interviews were recorded, anonymised and transcribed to be analysed by the researchers using a thematic analysis.

\subsection{Community Engagement Activities}

Our community engagement activities were conducted in four main localities, with participants recruited through partner organizations or existing relationships with each community. Participants were primarily mothers, with a small sampling of fathers and other caregivers. Our original intention was to start in-person ideation workshops including the community members and other stakeholders. However, due to the pandemic, we shifted to an online workshop followed by interviews with individual participants.

The workshop took place on Zoom, with the first hour primarily focused on academic discussions, and the second half taking place as a group discussion with partners and community members touching upon our initial ideas for digital Maternal and Child Health $(\mathrm{MCH})$ priorities as well as our plans for community engagement interviews. We set up each community in a separate breakout room, including 1-2 community members, 1-2 partner organization representatives, and 3-4 other researchers from varying disciplines. In two cases community members were given data bundles to cover the cost of connecting using their own mobile data connection. In one case, the community member attended from a local community center, facilitated by the local partner. In the third case, researchers travelled to the villages, and a small number of people gathered to attend the workshop together via a mobile data connection on the researchers' laptops.

Following this workshop, we conducted 30 interviews. This included eight participants from Cape Town, twelve from Limpopo and ten from Sweetwaters. We conducted interviews in the local language, through the use of mainly mobile or WhatsApp calls. However, due to poor network coverage and mixed access to devices, the interviews in rural Limpopo were conducted in person, with strict social distancing and the permission of local and institutional authorities. In-person research was not allowed by the institutions overseeing the work in Cape Town and Sweetwaters. Verbal consent was obtained at the beginning of every call and the calls were recorded. The recordings were then uploaded to a shared and secured data cloud, electronically transcribed and later analysed by a pool of five researchers using thematic analysis. In this paper we report on the process followed when conducting these interviews and researcher's experiences rather than the data gathered during the interviews.

\section{LESSONS LEARNED}

We reflect on our experiences conducting an online workshop, online interviews and on the recommendations provided by researchers and practitioners during the scoping and practice review.

\subsection{Including Community Members in Workshops}

While it was beneficial to include the community members in the workshop, we decided ultimately that the benefit did not outweigh the cost. Rural Limpopo has poor network coverage. This ultimately negated the benefit of including the participants in the workshop as they could not hear or be heard on the call and subsequently did not answer any of the researchers' questions. An alternative strategy would have been to transport the participants to a more urban location with better network. However, we felt it would be unsafe to transport a large number of people to an area with potentially higher infection rates.

Power differentials and socio-economic differences also played a significant role in the workshop. Not only were the community members outnumbered, but they were intimidated by many researchers from different disciplines who were all very eager to ask questions. At the same time, one particular individual (both a mother and a nurse) was confident in sharing her experiences, and both mothers were grateful to receive data bundles to participate.

\subsection{Online Interviewing with Community Members}

Conducting interviews online made it difficult to build trust and empathy as our first encounter with the participants was through a WhatsApp message confirming their participation in the study. The presence of power differentials complicated the interactions with the participants, specifically during the verbal informed consent process at the beginning of each interview. It was difficult to balance out these differentials as the process, not being in-person, felt too formal and intimidating for the participants. This was not conducive to creating a space for open conversations. We thus had to pay extra attention to building empathy as many of the questions asked could trigger distress and/or unpleasant memories. This was further complicated by unstable connections that would often drop or degrade the quality of the call which forced participants to repeat their often emotional answers. In addition, the interviews were often interrupted by family responsibilities or had to be rescheduled for a later date because it was particularly hard for mothers to stick to time slots when they had to care for their infants.This stop start nature of the interviews was challenging for the both 
the researchers and participants as the process often had to be restarted. Participant recruitment was also difficult at times as some participants did not reply to the WhatsApp invitations, resulting in the researcher making a maximum of two follow-up calls before deciding to remove the participant from the list.

\subsection{Researcher and Practitioner Recommendations to Foster Community Engagement}

The researchers provided recommendations to consider when conducting research with vulnerable communities. This included combining synchronous technologies such as interviews and focus groups conducted via video conferencing platforms or online collaboration tools such as Miro with asynchronous tools and methods such as diaries, journals, activity books, probes. and etc.

We can still send things on the Mail and if that's necessary and that can be technology, or it can be something else that's perhaps a little bit more creative and engaging. So without the expectation I have [of] having them collaborate on the screen on line on a call, but doing something more like probe-based -Researcher

It would be when I would use something like Miro for example, which is this collaborative online Software where we can draw in paint and write and whatnot. Which I'm sure it still novel so it feels engaging -Practitioner

However, a blend of synchronous and asynchronous technologies might be overwhelming, especially when dealing with participants who are not comfortable with technology. Technology champions (a person comfortable with navigating online collaboration tools and technology) can assist in these instances by enabling the participants to partake in the discussions. This champion can translate discussions into the online collaboration technology using the voice of the participants. We should carefully consider that not all participants are equal in terms of Internet access and using computing devices. It is therefore recommended that researchers make use of a wide range of technology and scaffolding methods according to the needs of the participants with many studies employing video conferencing software where it can be used, then reverting to WhatsApp calls where video conferencing is not possible and finally using voice notes and SMS as the access to stable Internet connections and smart phones decreases.

We made sure that our kind of animations or videos are small enough to be able to be shared via WhatsApp. We have also worked with another organization, too create very low tech approach is translating a lot of that those scripts into kind of interactive voice response type script so that is available for Community health workers in areas that we really only have feature phones -Practitioner

One final contribution from our interviews was that we should not discount traditional methods, as they might still work in an online setting with some tweaking and retrofitting for online. For example, making use of conference calls, online collaboration tools and paper during a workshop to enable all participants in the session to contribute. Participants who are not fully comfortable with technology would then be able to take pictures of their designs, share it with the workshop and have it uploaded to the common work space.

\section{DISCUSSION}

\subsection{Technical Feasibility}

From our experiences we have learned that synchronous connections and other technology based connections are not always possible when considering vulnerable communities. As it was the case with the inclusion of community members in an online workshop where the members from rural Limpopo were unable to join and subsequently participate in the call. It is thus necessary to consider the technical feasibility of any study aimed at these communities. Workarounds like transporting community members to a central location with good Internet access when it is safe to do so, making use of WhatsApp[5] calls and purchasing data bundles can be applied to remedy these issues.

\subsection{Building Empathy and Dealing with Power Imbalances}

Building empathy and addressing power imbalances can to be difficult in in-person research with many researchers paying special attention to this area. This is amplified when an interview or workshop is taken into the online space [12]. Researchers should be prepared for the fact that they will dedicate more time to gain the trust of their participants. They should also consider the fact that they may not be able to build enough trust, and thus consider the influence of these amplified power imbalances on the responses given and he quality of the data.

\subsection{Basket of Methods}

Finally, researchers need to be prepared with a variety of options, both in terms of technology and online/offline methods used, to respond to technical difficulties as they arise [3]. This basket of methods is further useful when one considers the fact that not all resource constraint communities are equal, constraints can differ within research contexts such as geographical area even between participants [9]. Additional planning needs to be in place when using online collaborative tools as not all the participants, even fellow researchers, would have the same digital expertise and might not be able to effectively participate during the active sessions. Employing technology champions and moderators who can assist or even complete the online activity following the feedback and voice of the participants, have proven to be beneficial. Last but not least, flexibility is key as interviews are often rescheduled due connectivity issues and unexpected family emergencies.

\section{CONCLUSION AND FUTURE WORK}

Many research methods for conducting fieldwork during pandemics exist [8], however, careful consideration should be given when choosing methods for low-resource constraint communities, especially in Low to Middle Income Countries (LMICs) as additional challenges would emerge in situ. These communities exist in areas with poor Internet connectivity, with community members often only owning feature phones and needing to travel to different 
locations to participate in a study. Researchers should consider these challenges and carefully select their research methods, also considering that they might need to change and/or adapt their methods and tools not only between communities, but also within a single community, as access to devices and Internet connectivity varies between participants. Finally, combining synchronous and asynchronous tools has proven to be successful and useful when conducting research with vulnerable communities. Future work should focus on more appropriate methods considering the caveats of these communities.

\section{ACKNOWLEDGMENTS}

We would like to thank all the participants in the study including the CoMaCH UK-South Africa Network members as well as local and international partners, cross-disciplinary co-researchers as well as practitioners with experience engaging with communities. This study was funded by the UKRI GCRF Digital Innovation for Development in Africa (DIDA) awards (Ref: EP/T030429/1.).

\section{REFERENCES}

[1] Joal D. Beane, Priya H. Dedhia, Aslam Ejaz, Carlo M. Contreras, Jordan M. Cloyd, Allan Tsung, and Timothy M. Pawlik. 2020. Conducting Clinical Trials in the Time of a Pandemic. Annals of Surgery 272, 3 (Sept. 2020), e219. https://doi.org/ 10.1097/SLA.000000000004114 Publisher: Wolters Kluwer Health.

[2] James Brian Byrd, Natalie Bello, and Michelle N. Meyer. 2020. Pandemic Pandemonium. Circulation (June 2020). https://doi.org/10.1161/CIRCULATIONAHA. 120.047347 Publisher: Lippincott Williams \& Wilkins Hagerstown, MD.

[3] Melissa Densmore, Ben Bellows, John Chuang, and Eric Brewer. 2013. The Evolving Braid: How an Organization in Uganda Achieved Reliable Communications.
In Proceedings of the Sixth International Conference on Information and Communication Technologies and Development: Full Papers - Volume 1 (Cape Town, South Africa) (ICTD '13). Association for Computing Machinery, New York, NY, USA, 257-266. https://doi.org/10.1145/2516604.2516620

[4] Yogesh K. Dwivedi, D. Laurie Hughes, Crispin Coombs, Ioanna Constantiou, Yanqing Duan, John S. Edwards, Babita Gupta, Banita Lal, Santosh Misra, Prakhar Prashant, Ramakrishnan Raman, Nripendra P. Rana, Sujeet K. Sharma, and Nitin Upadhyay. 2020. Impact of COVID-19 pandemic on information management research and practice: Transforming education, work and life. International fournal of Information Management 55 (Dec. 2020), 102211. https://doi.org/10. 1016/j.ijinfomgt.2020.102211

[5] Kerry Gibson. 2020. Bridging the digital divide: Reflections on using WhatsApp instant messenger interviews in youth research. Qualitative Research in Psychology 0, 0 (2020), 1-21. https://doi.org/10.1080/14780887.2020.1751902 arXiv:https://doi.org/10.1080/14780887.2020.1751902

[6] Monique Hennink, Inge Hutter, and Ajay Bailey. 2020. Qualitative Research Methods. SAGE.

[7] Marnie Howlett. 2021. Looking at the 'field' through a Zoom lens: Methodological reflections on conducting online research during a global pandemic:. Qualitative Research (Jan. 2021). https://doi.org/10.1177/1468794120985691 Publisher: SAGE PublicationsSage UK: London, England.

[8] D Lupton (ed.). 2020. Doing Fieldwork in a Pandemic (crowdsourced document). https://docs.google.com/document/d/ 1clGjGABB2h2qbduTgfqribHmog9B6P0NvMgVuiHZCl8/edit?ts=5e88ae0a\#

[9] Segametsi Molawa. 2009. The "first" and "third world" in Africa: knowledge access, challenges and current technological innovations in Africa. (2009).

[10] Mirella Mourad, Sabine Bousleiman, Ronald Wapner, and Cynthia GyamfiBannerman. 2020. Conducting research during the COVID-19 pandemic. Seminars in Perinatology 44, 7 (2020), 151287. https://doi.org/10.1016/j.semperi.2020.151287 COVID Preparedness: Lessons Learned from the Pandemic Center: Issue 2.

[11] Alison Castro Superfine. 2020. Conducting research in the time of pandemic: A pause or an opportunity? Journal of Mathematics Teacher Education 23, 5 (Oct. 2020), 429-431. https://doi.org/10.1007/s10857-020-09478-w

[12] Kentaro Toyama. 2011. Technology as Amplifier in International Development. In Proceedings of the 2011 IConference (Seattle, Washington, USA) (iConference '11). Association for Computing Machinery, New York, NY, USA, 75-82. https: //doi.org/10.1145/1940761.1940772 